Check for updates

Cite this: RSC Adv., 2017, 7, 41667

Received 27th June 2017

Accepted 18th August 2017

DOI: $10.1039 / c 7 r a 07118 c$

rsc.li/rsc-advances

\section{Transformation and dehydration kinetics of methylene blue hydrates detected by terahertz time-domain spectroscopy $\dagger$}

\author{
Shihan Yan, $t^{a}$ Hua Zhang, $t^{a}$ Zhongbo Yang, ${ }^{a}$ Mingjie Tang, ${ }^{a}$ Mingkun Zhang, ${ }^{a}$ \\ Chunlei Du, ${ }^{a}$ Hong-Liang Cui ${ }^{\mathrm{ab}}$ and Dongshan Wei $\mathbb{D}^{* a}$
}

\begin{abstract}
Methylene blue (MB) as an important dye and medicine, has several different crystalline hydrates. These MB hydrates can transform from one to another at different temperatures or humidities. Determination of these hydrates is a big challenge for traditional spectroscopic technologies like infrared spectroscopy (IR) and Raman spectroscopy due to the component and structure similarities among these hydrates. We present a terahertz ( $\mathrm{THz}$ ) spectroscopy technology to differentiate three typical MB hydrates: pentahydrate, dihydrate and anhydrate. The pentahydrate exhibits prominent $\mathrm{THz}$ absorption features at 0.84 and $1.68 \mathrm{THz}$, and the dihydrate exhibits a broad and weak absorption peak at $0.89 \mathrm{THz}$, while the anhydrate has no obvious characteristic absorption peak at the measured spectral range of $0.2-2.0 \mathrm{THz}$. The dehydration kinetics of $\mathrm{MB}$ hydrates is investigated according to the variation of one of the main $\mathrm{THz}$ characteristic absorption peaks of $M B$ pentahydrate with the heating time at different heating temperatures. A clear relation between the dehydration rate and the heating temperature can be fitted by an Arrhenius equation. The fitted activation energy of $64.5 \mathrm{~kJ} \mathrm{~mol}^{-1}$ is quite consistent with the enthalpy change due to the transformation of MB pentahydrate to $M B$ anhydrate reported in a previous study.
\end{abstract}

\section{Introduction}

Methylene blue (MB) was first prepared in 1876 by Heinrich Caro, and it is a heterocyclic aromatic chemical compound with a chemical formula of $\mathrm{C}_{16} \mathrm{H}_{18} \mathrm{~N}_{3} \mathrm{SCl}$ (see Fig. 1). It has been widely used in chemical and biological fields..$^{1-5}$ For example, it can be used as pharmaceutical drugs, chemical indicator and biological stain. Methylene blue has been described as "the first fully synthetic drug used in medicine". It is a component of

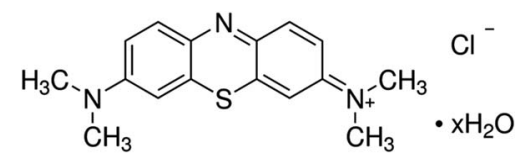

Fig. 1 Molecular structures of MB hydrates.

\footnotetext{
${ }^{a}$ Chongqing Key Laboratory of Multi-Scale Manufacturing Technology, Chongqing Institute of Green and Intelligent Technology, Chinese Academy of Sciences, Chongqing, 400714, China. E-mail: dswei@cigit.ac.cn

${ }^{b}$ College of Instrumentation Science and Electrical Engineering, Jilin University, Changchun, Jilin, 130061, China

$\dagger$ Electronic supplementary information (ESI) available. See DOI: $10.1039 / \mathrm{c} 7 \mathrm{ra0} 07118 \mathrm{c}$

$\ddagger$ These authors contributed equally to this work.
}

frequently prescribed urinary analgesic/anti-infective/antispasmodic known as "Prosed DS". Due to the existing strong hydrogen-bond interaction between $\mathrm{MB}$ and water, $\mathrm{MB}$ can easily form several stable hydrated crystalline states with different water contents at room temperature and at different humidities. ${ }^{6}$ Researches have shown that different crystalline forms of medicines can affect the stability, homogeneity, active pharmaceutical ingredient (API) and safety of the medicines to some degree. ${ }^{7,8}$ Therefore, it is significant to monitor the crystalline states of methylene blue during its applications.

A number of techniques have been used to test the crystal forms of medicines, such as X-ray diffraction (XRD), ${ }^{7,8}$ Infrared (IR) ${ }^{9,10}$ Raman, ${ }^{10,11}$ circular dichroism ${ }^{12}$ spectra, differential scanning calorimetry (DSC), ${ }^{13}$ thermogravimetric analysis (TGA), ${ }^{14}$ differential thermal analysis (DTA), ${ }^{15}$ solid-state nuclear magnetic resonance, ${ }^{16}$ magnetic anisotropy, ${ }^{17}$ dilatometer and polarizing microscope.$^{18}$ However, each individual technique has its own limitation ${ }^{19}$ and usually need two or more techniques combined to study the crystalline states of hydrates. Also, the technique should be applicable to the online processing and manufacture if it is the ultimate intention to use the technique for process optimization. XRD is the standard method for researching crystallinity, ${ }^{20}$ but in order to get accurate crystal structure data, the complicated single-crystal preparation and prudent crystal structure analysis are requisite. Furthermore, the positions of hydrogen atoms cannot be 
precisely determined by the XRD technique. ${ }^{21}$ The emerging requirement on process analytical technologies (PAT) has led to an increasing utilization of optical spectroscopy technologies such as IR and Raman which can potentially perform online analyses. ${ }^{22}$ However, since IR and Raman spectroscopies mainly characterize the intra-molecular vibrations including chemical bonds and atomic groups, it is difficult for them to differentiate crystalline hydrates with different water contents.

Terahertz (THz) wave lying between the infrared and microwave regions of the electromagnetic waves, has received considerable attention because of its potentially important applications. $^{23,24} \mathrm{THz}$ spectroscopy is capable of characterizing the intra- and inter-molecular vibrations. The unique fingerprints of crystalline compounds at $\mathrm{THz}$ frequencies, mainly originating from the inter-molecular low-frequency coherent motions, make terahertz spectroscopy a very sensitive technique in differentiating crystalline polymorphs, ${ }^{25}$ hydrates ${ }^{26-30}$ and cocrystals ${ }^{31-33}$ and also allow the observation of phase transformations between solid state modifications. ${ }^{34}$ Terahertz time-domain spectroscopy (THz-TDS), as a relatively new technology, has been developed to study inter-molecular vibrational modes, ${ }^{35,36}$ hydrogen-bond stretching, van der Waals interaction, ${ }^{26,31,37}$ and torsion vibrations in many chemical and biological compounds, including small biomolecules, pharmaceutical materials, and explosives, ${ }^{38,39}$ to explore the conformational and structural dynamics of biomolecules, ${ }^{40,41}$ to identify isomers of amino acids such as L-, D-, and DL-alanine, L-serine and DL-serine, $\alpha$ - and $\gamma$-glycine ${ }^{\mathbf{4 2 - 4 4}}$ and to investigate the dehydration kinetics of crystalline hydrates. ${ }^{28,29}$ The dehydration kinetics of crystalline hydrates could be investigated by analyzing the changes of their terahertz spectra under different states and reaction processes.

In this work, the $\mathrm{MB}$ pentahydrate is first purchased and two other stable hydrates of MB dihydrate and MB anhydrate are prepared from the pentahydrate in experiment. THz spectroscopy technology is then presented to differentiate these three MB hydrates. For comparison, the IR and Raman spectroscopy measurements are also performed. Lastly, the dehydration kinetics of the MB pentahydrate transforming into MB anhydrate is investigated according to the variation of one of the characteristic $\mathrm{THz}$ absorption peaks of $\mathrm{MB}$ pentahydrate with the heating time. This work clearly illustrates the advantages of THz spectroscopy in differentiating the crystalline hydrates and investigating the dehydration kinetics.

\section{Experimental}

\subsection{Chemicals and materials}

Methylene blue pentahydrate (CAS-number 7220-79-3, Fig. 1) was purchased from Aladdin (Shanghai, China) in HPLC grade and used without further purification. All solvents were purchased from SCR (Shanghai, China) in analytical grade and were used as received without further purification.

\subsection{Sample preparation}

Firstly, the three methylene blue hydrates were prepared referring to the reported recrystallization method. ${ }^{6} \mathrm{MB}$ pentahydrate was obtained by recrystallization in $\mathrm{HCl}$ aqueous. $\mathrm{MB}$ dihydrate was prepared by a suspension equilibration of the pentahydrate in a 10 -fold excess of 2 - $\mathrm{PrOH}$ with $0.5 \% \mathrm{H}_{2} \mathrm{O}$ and dried in an oven at $40{ }^{\circ} \mathrm{C}$ for $3 \mathrm{~h}$. $\mathrm{MB}$ anhydrate was directly from the product of the pentahydrate dried at $60{ }^{\circ} \mathrm{C}$ for overnight. For subsequent measurements, these $\mathrm{MB}$ hydrates were grinded into powder by using agate mortar and then the pure powders were pressed into pallets with a diameter of $15.0 \mathrm{~mm}$ and a thickness of around $0.5 \mathrm{~mm}$ using a manual tablet press. Crystalline structures of these three MB hydrates were analyzed by X-ray powder diffraction patterns (XRPD) (Fig. S1 in the ESI $\dagger$ ) and their water contents were determined by thermogravimetric analysis (TGA) (Fig. S2 in ESI $\dagger$ ). From Fig. S1, $\uparrow$ it can be seen that there is prominent difference between the XRPD patterns of the three MB hydrates due to their different crystal structures. In addition, the X-ray diffraction intensity from the MB pentahydrate is obviously higher than those of MB dehydrate and anhydrate, indicating the crystallinity of the former is better than the latter two ones.

XRPD patterns of the samples were recorded with PANalytical (Holland) X'Pert3 Powder. Measurements were performed with $\mathrm{Cu} \mathrm{K} \alpha$ radiation $(\lambda \approx 0.154 \mathrm{~nm})$ at $40 \mathrm{kV} / 40 \mathrm{~mA}$. Data points were collected with a step size of $0.02^{\circ}$ in $2 \theta$ and an accumulation time of $37 \mathrm{~s}$ per step. The samples were prepared on a glass holder with a depth of $1.0 \mathrm{~mm}$ and a diameter of 12 $\mathrm{mm}$. All samples were rotated at a speed of $30 \mathrm{rpm}$ during the XRPD measurement.

The TGA analysis was performed using a thermobalance TGA/DSC from METTLER-TOLEDO Instruments (Switzerland). The samples were placed in a ceramic crucible and heated up in

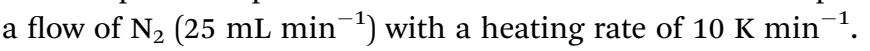

\subsection{Attenuated total reflection infrared spectroscopy}

Infrared spectra were measured by a Agilent Cary 630 FTIR Spectrometer (Agilent, The United States) equipped with a single reflection diamond attenuated total reflectance (ATR) accessory, which did not require sample preparation. A highquality spectrum was obtained simply by placing the sample on a diamond sensor, and pressing the sample to ensure good contact. Spectral data were recorded in a wavenumber range from 4000 to $400 \mathrm{~cm}^{-1}$ with a resolution of $2 \mathrm{~cm}^{-1}$ and an average over 100 scans.

\subsection{Raman spectroscopy}

Raman measurements were performed by using a Renishaw (The United Kingdom) inVia micro-Raman spectroscopy system, equipped with three laser excitations (532 nm DPSS laser, $50 \mathrm{~mW}$; $633 \mathrm{~nm}$ He-Ne laser, $17 \mathrm{~mW} ; 785 \mathrm{~nm}$ diode laser, $250 \mathrm{~mW}$ ). A Leica microscope with $50 \times$ objective was employed to focus the incident laser on the samples for collection of back-scattered Raman signals. Before the Raman spectra were measured, the wavenumber of the Raman band of silicon at $520 \mathrm{~cm}^{-1}$ was calibrated, and all data were collected under the same conditions. The spectral range was from 400 to $3000 \mathrm{~cm}^{-1}$, the acquisition time of each spectrum was fixed at $10 \mathrm{~s}$, and power on the sample was $0.05 \%$ of the laser power using $532 \mathrm{~nm}$ DPSS laser. 


\subsection{THz spectroscopy}

THz spectroscopy measurements were performed using a Picometrix T-ray 5000 fiber-coupled spectrometer (Advanced Photonix, Inc., MI, USA) in transmission mode. The spectrometer used femtosecond near-infrared laser pulses and LT-InGaAs photoconductive antenna (PCA) chips to generate and coherently detect the electric field of ultrashort $\mathrm{THz}$ electromagnetic pulses in the time domain. All of $\mathrm{THz}$ spectroscopy measurements were performed at a relative humidity of $<2.0 \%$ with the purge of nitrogen gas. The room temperature measurements were conducted at an ambient temperature of $21.0 \pm 0.4{ }^{\circ} \mathrm{C}$. For the variable temperature measurements, to control the temperature, a variable temperature cell holder (Specac Inc., Orpington, UK) was used and the testing temperature range was from 20 to $160{ }^{\circ} \mathrm{C}$ with an accuracy of $\pm 0.2{ }^{\circ} \mathrm{C}$. The heating rate was $10{ }^{\circ} \mathrm{C} \min ^{-1}$ for the temperature varying from $20^{\circ} \mathrm{C}$ to $160{ }^{\circ} \mathrm{C}$. For the dehydration kinetics measurement of the pentahydrate, all of the heating rates were the same to be $15{ }^{\circ} \mathrm{C} \min ^{-1}$ for the sample temperature increasing from $20{ }^{\circ} \mathrm{C}$ to the target temperatures of $45{ }^{\circ} \mathrm{C}, 50{ }^{\circ} \mathrm{C}, 55{ }^{\circ} \mathrm{C}, 60{ }^{\circ} \mathrm{C}$ and $75{ }^{\circ} \mathrm{C}$, respectively. After reaching the target temperature, heating was maintained for about 40 minutes at each target temperature to observe the dehydration process.

$\mathrm{THz}$ time-domain waveforms of the sample and the reference without sample (air) were measured under the same experimental conditions. The frequency-domain spectra of measured signals were obtained by Fourier transform (FT). The effective frequency region is 0.2 to $2.0 \mathrm{THz}$ for THz-TDS measurements. Spectral frequency resolution of the spectrometer is $12.5 \mathrm{GHz}$. Measurements were performed three times at different days for each sample to eliminate the influence of the instrument performance.

The THz optical refractive index of the MB sample is calculated using the equation ${ }^{\mathbf{4 5 , 4 6}}$

$$
n(\omega)=\frac{\left|\varphi_{\mathrm{s}}(\omega)-\varphi_{\text {ref }}(\omega)\right| c}{2 \pi \omega d}+1
$$

where $\varphi_{\mathrm{s}}(\omega), \varphi_{\text {ref }}(\omega)$ are the phase angles of the Fourier transforms of the power transmissions of the MB sample, $I_{\mathrm{s}}$, and the reference (air), $I_{\text {ref }}$, respectively, and $c$ the light speed, $\omega$ the frequency and $d$ the thickness of the MB sample. For the solid measurement, the absorption coefficient of a free-standing sample is calculated as $^{\mathbf{4 6}}$

$$
\alpha(\omega)=\frac{2}{d} \ln \left[\frac{4 n(\omega)}{\rho(\omega)[n(\omega)+1]^{2}}\right]
$$

where $\rho(\omega)$ is the amplitude ratio of the Fourier transforms of $I_{\mathrm{s}}$ and $I_{\text {ref. }}$.

\section{Results and discussions}

\subsection{IR and Raman characterization}

The ATR-FTIR and the Raman spectra of these three MB hydrates are shown in Fig. 2 and 3, respectively. From Fig. 2 we can see that there are obvious spectral differences around 2000 and $3300 \mathrm{~cm}^{-1}$ between the MB pentahydrate or dihydrate and

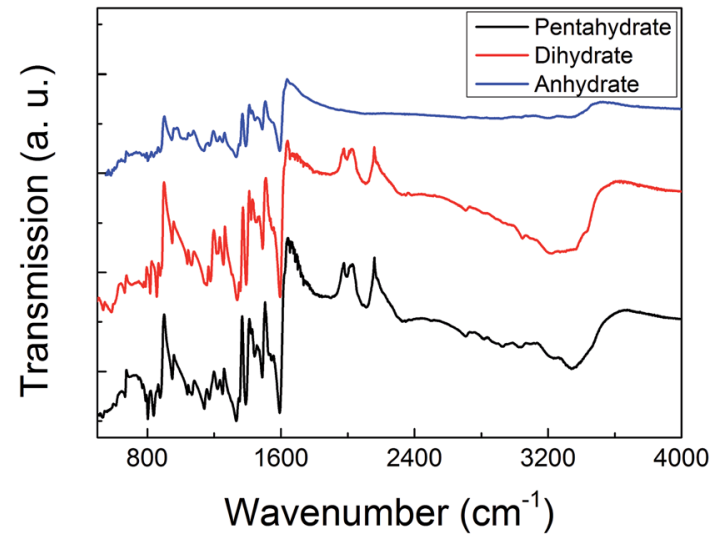

Fig. 2 ATR-IR spectra of MB pentahydrate, dihydrate and anhydrate.

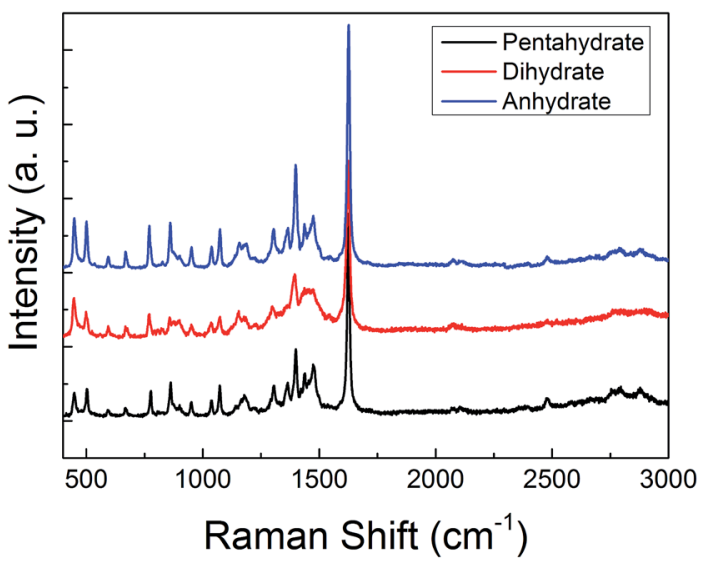

Fig. 3 Raman spectra of MB pentahydrate, dihydrate and anhydrate.

the $\mathrm{MB}$ anhydrate due to the absence of water in the $\mathrm{MB}$ anhydrate. While the difference in ATR-FTIR spectra observed between the pentahydrate and the dihydrate are much less pronounced, which leads to the feasibility to identify MB hydrates by infrared spectroscopy is very limited.

As for the Raman measurements between 400 and $3000 \mathrm{~cm}^{-1}$, since the Raman spectroscopy is not sensitive to water, there is no obvious difference in Raman vibration modes between all of these three hydrates (see Fig. 3). Therefore, it would be difficult to make a distinction between MB hydrates by Raman spectroscopy at this frequency range of $400-3000 \mathrm{~cm}^{-1}$. We notice that the recently appeared THz-Raman spectroscopy system which extends the traditional Raman spectroscopy into the THz-frequencies (lower than $200 \mathrm{~cm}^{-1}$ ) has been used to investigate the low-frequency lattice vibrations of pharmaceutical molecules and their amorphous-crystalline transformations. ${ }^{47,48}$ It is interesting to use this THz-Raman spectroscopy system to detect MB hydrates and their transformation in future.

\subsection{THz spectroscopy characterization}

To test the capability of $\mathrm{THz}$ spectroscopy in differentiating these hydrates, we measured the $\mathrm{THz}$ absorption spectra of 

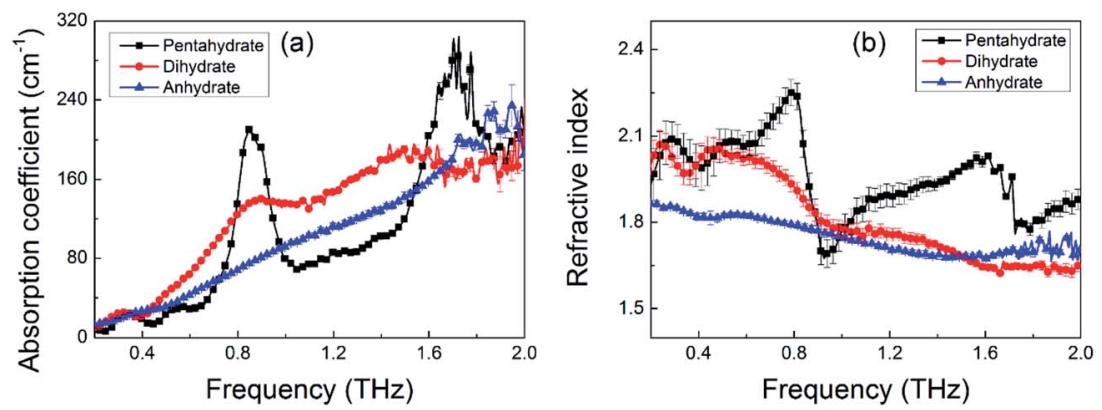

Fig. 4 THz spectra of MB pentahydrate, dihydrate and anhydrate. (a) Absorption coefficient; (b) refractive index.

these three MB hydrates. The room temperature THz absorption spectra of these three MB hydrates from 0.2 to $2.0 \mathrm{THz}$ are shown in Fig. 4a. For MB pentahydrate, there are four absorption peaks at $0.36,0.54,0.84$, and $1.68 \mathrm{THz}$ and the latter two absorption peaks are much stronger than the former two ones. For MB dihydrate, a broad and weak absorption peak appears at $0.89 \mathrm{THz}$ and another two weaker absorption peaks at 0.31 and $1.50 \mathrm{THz}$ could also be found with careful observation. While for the MB anhydrate, there is no obvious absorption peak at the measured frequency range. The featuring $\mathrm{THz}$ absorption of these hydrates showed in Fig. 4a is associated with the crystalline states of the MB molecules. Such difference in $\mathrm{THz}$ absorption spectra caused by crystalline water had also been observed in the THz spectra of the monohydrated and anhydrous glucose. ${ }^{28,29}$ In addition, there are also notable difference in the refractive index between these three MB hydrates as shown in Fig. 4b. These results demonstrated the advantage of $\mathrm{THz}$ spectroscopy in molecular identification, especially for crystalline hydrates.

Although XRD can differentiate these three MB hydrates seen from Fig. S1† due to the crystal structure difference between these hydrates, for the XRD measurement, there is a prerequisite that the measured sample has a good crystallinity, which will limit the detection of non-crystalline samples. While the THz spectroscopy technique does not have the limit and it can not only detect the crystalline samples, ${ }^{49}$ but also the amorphous samples including biological solutions ${ }^{50}$ and glass-state materials ${ }^{51}$ in a non-destructive way and without complicated sample preparation.

It is worth noting that in the present work, we only show THz spectroscopy technique is capable of detecting MB hydrates from the single or pure sample. As for the unknown sample, especially for the mixture detection in practice, once the $\mathrm{THz}$ spectrum of its each component is obtained, the unknown or mixture sample can be determined by combining the $\mathrm{THz}$ spectroscopy and the chemometric analysis. ${ }^{52,53}$

To observe the temperature effect on the $\mathrm{THz}$ spectrum of the MB hydrate, Fig. 5 displays the $\mathrm{THz}$ absorption spectra of the MB pentahydrate heated from $20{ }^{\circ} \mathrm{C}$ to $160^{\circ} \mathrm{C}$ at a heating rate of $10^{\circ} \mathrm{C}$ per minute. With the elevation of the temperature, the intensities of the absorption peaks of MB pentahydrate at 0.84 and $1.68 \mathrm{THz}$ decrease gradually and at the temperature higher than $75{ }^{\circ} \mathrm{C}$, both of these absorption peaks begin to disappear, which indicates that crystalline state of MB pentahydrate changes upon heating. Furthermore, the positions of these absorption peaks shifted slightly to lower frequencies from $20^{\circ} \mathrm{C}$ to $75{ }^{\circ} \mathrm{C}$ as shown in Fig. $5 \mathrm{~b}$. These shifts arise from the temperature dependence of the vibrational modes of the $\mathrm{MB}$ pentahydrate crystal due to the anharmonicity of vibrational potentials. ${ }^{28}$

\subsection{Dehydration kinetics of $\mathrm{MB}$ pentahydrate}

To investigate the dehydration kinetics, variations of $\mathrm{THz}$ absorption spectra of MB pentahydrate samples which kept
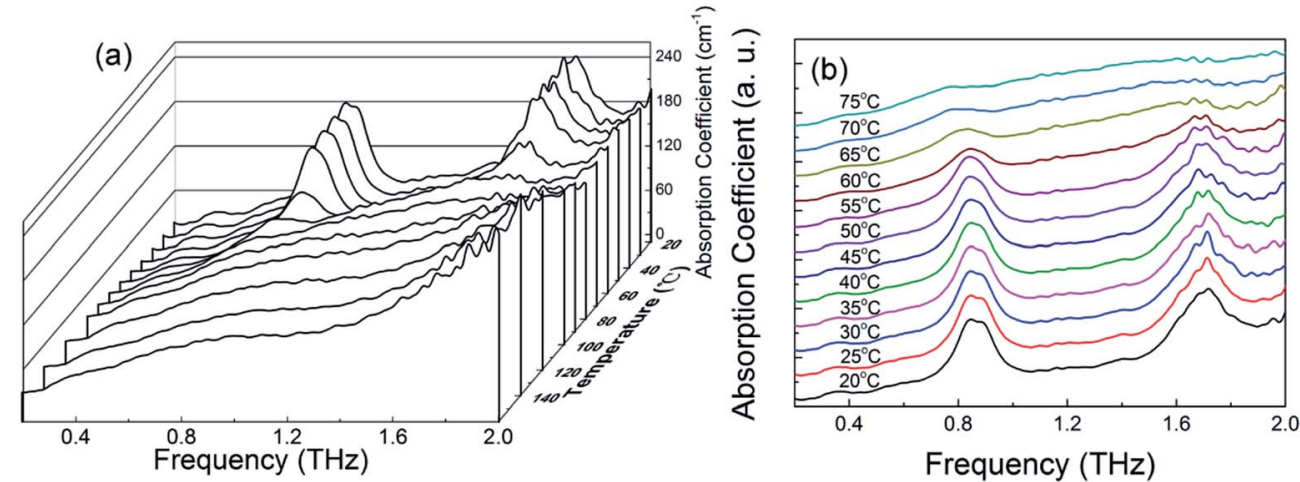

Fig. 5 (a) Variation of absorption coefficient of MB pentahydrate with frequency at $20-160^{\circ} \mathrm{C}$; (b) THz absorption spectra of MB pentahydrate at $20-75{ }^{\circ} \mathrm{C}$. 
to be heated at $45,50,55,60$ and $75{ }^{\circ} \mathrm{C}$ with heating time at 0.7-1.0 THz were studied and shown in Fig. 6a-e, respectively. We also checked the variation of $\mathrm{THz}$ spectra around the absorption peak at $1.68 \mathrm{THz}$. However, since the decreased signal-to-noise ratio accompanied by the increased frequency reduced the accuracy in quantitative analysis of the dehydration kinetics, we focused on the variation of the characteristic absorption peak at lower frequency of 0.84 THz. When the MB pentahydrate sample keeps heating, the absorption peak area between 0.7 and $1.0 \mathrm{THz}$ decreases continuously with the heating time until zero at each heating temperature as shown in Fig. 6f, which indicates the MB pentahydrate has experienced a transformation into $\mathrm{MB}$ anhydrate. As seen from this figure, higher temperatures brought faster rates of losing crystalline water. The relation between the normalized absorption peak area and the heating time was linearly fitted and the dehydration rates at different heating temperatures were obtained from the fittings.

In line with the general Arrhenius equation, the dehydration rate, $k$, is related with the heating temperature as

$$
k=A \exp \left(-E_{\mathrm{A}} / R T\right)
$$

where $A$ is a prefactor, $E_{\mathrm{A}}$ is the activation energy $\left(\mathrm{kJ} \mathrm{mol}^{-1}\right), R$ is the ideal gas constant $\left(8.314 \mathrm{~J} \mathrm{~mol}^{-1} \mathrm{~K}^{-1}\right)$, and $T$ is the heating temperature in unit of $\mathrm{K}$. Using this equation, the activation energy of the dehydration of $\mathrm{MB}$ pentahydrate transforming into $\mathrm{MB}$ anhydrate was derived to be $64.5 \mathrm{~kJ} \mathrm{~mol}^{-1}$ according to the linear fitting result illustrated in Fig. 7, which is in good agreement with the reported result of $60.9 \mathrm{~kJ} \mathrm{~mol}^{-1}$ by the solution calorimetry and DCS measurement. ${ }^{6}$ Therefore, the present investigation indicates that THz-TDS is an accurate and effective technique to characterize the dehydration kinetics of crystalline hydrates.
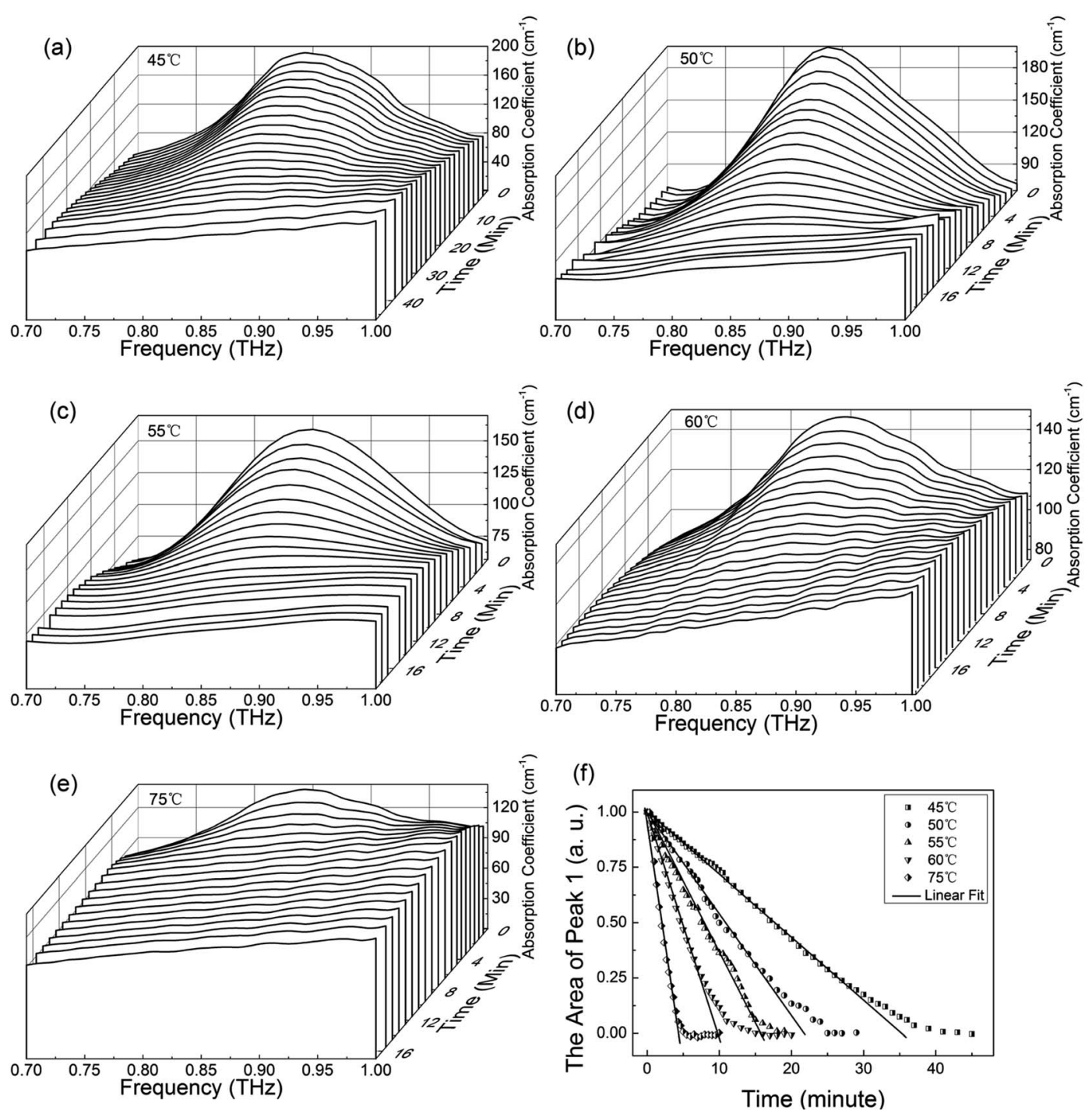

Fig. $6 \mathrm{THz}$ absorption spectra of $\mathrm{MB}$ pentahydrate at $0.7-1.0 \mathrm{THz}$ heated at $45^{\circ} \mathrm{C}$ (a), $50{ }^{\circ} \mathrm{C}$ (b), $55^{\circ} \mathrm{C}$ (c), $60^{\circ} \mathrm{C}$ (d) and $75^{\circ} \mathrm{C}$ (e) with the same heating rate of $15^{\circ} \mathrm{C} \mathrm{min}{ }^{-1}$ and variation of the normalized absorption peak area at $0.7-1.0 \mathrm{THz}$ with the heating time at the different heated temperatures (f). 


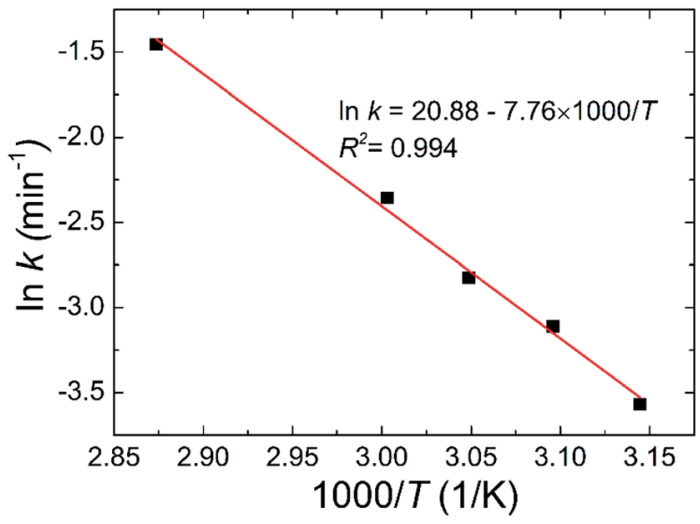

Fig. 7 Plot of $\ln k$ vs. 1000/T. The scatter symbols are the experimental data and the solid.

\section{Conclusions}

The discovery of pharmaceutical application of methylene blue has given renewed impetus to studies on the crystalline states of this compound. The presented novel study on the crystalline transformation of methylene blue due to the temperature change by $\mathrm{THz}$ spectroscopy had led to the identification of three different hydrates with clearly distinct spectral features. The crystalline state changes of MB hydrates during the heating process was confirmed through the observation of the changes of characteristic $\mathrm{THz}$ absorption peaks. These continuous changes were analyzed and used to determine the transformation (dehydration) kinetics of methylene blue at different temperatures.

To compare with the $\mathrm{THz}$ technique, the detection capability of IR and Raman spectroscopy techniques is limited since the MB hydrates (penta- and di-hydrates) have the same functional groups. Although the TGA and the XRD methods may also be used to detect the crystalline states of hydrates as shown in this work, the former needs to destroy the structure of crystalline hydrates upon heating and the latter needs the complicated crystal preparation. Therefore, $\mathrm{THz}$ spectroscopy technique is entitled with advantages in investigating the crystalline states of materials in a nondestructive and simple way and may help to monitor the manufacturing process, dosing, storage stability and bioavailability of medicinal substances.

\section{Conflicts of interest}

There are no conflicts of interest to declare.

\section{Acknowledgements}

This work is part supported by National 973 Program of China (No. 2015CB755401), the National Key Research and Development Program of China (2017YFF0106303, 2016YFC0101300 and 2016YFC0101002), and national natural science foundation of China (11504372, 21407145, 31400625 and 61605206).

\section{References}

1 M. Andresen, A. Dougnac, O. Diaz, G. Hernandez, L. Castillo, G. Bugedo, M. Alvarez and J. Dagnino, Use of methylene blue in patients with refractory septic shock: Impact on hemodynamics and gas exchange, J. Crit. Care, 1998, 13, 164-168.

2 S. C. Salaris and C. F. Babbs, Methylene blue as an inhibitor of superoxide generation by xanthine oxidase. A potential new drug for the attenuation of ischemia/reperfusion injury, Biochem. Pharmacol., 1991, 42, 499-506.

3 R. H. Schirmer, B. Coulibaly, A. Stich, M. Scheiwein, H. Merkle, J. Eubel, K. Becker, H. Becher, O. Muller, T. Zich, W. Schiek and B. Kouyate, Methylene blue as an antimalarial agent, Redox Rep., 2003, 8, 272-275.

4 J. P. Tardivo, A. Del Giglio, C. S. de Oliveira, D. S. Gabrielli, H. C. Junqueira, D. B. Tada, D. Severino, R. d. F. Turchiello and M. S. Baptista, Methylene blue in photodynamic therapy: From basic mechanisms to clinical applications, Photodiagn. Photodyn. Ther., 2005, 2, 175-191.

$5 \mathrm{~K}$. Taylor and H. Holtby, Methylene blue revisited: Management of hypotension in a pediatric patient with bacterial endocarditis, J. Thorac. Cardiovasc. Surg., 2005, 130, 566.

6 T. Rager, A. Geoffroy, R. Hilfiker and J. M. D. Storey, The crystalline state of methylene blue: a zoo of hydrates, Phys. Chem. Chem. Phys., 2012, 14, 8074-8082.

7 H.-Q. Li, J.-Y. Xue, L. Shi, S.-Y. Gui and H.-L. Zhu, Synthesis, crystal structure and antimicrobial activity of deoxybenzoin derivatives from genistein, Eur. J. Med. Chem., 2008, 43, 662-667.

8 K. Ohta, T. Goto, S. Fujii, M. Kawahata, A. Oda, S. Ohta, K. Yamaguchi, S. Hirono and Y. Endo, Crystal structure, docking study and structure-activity relationship of carborane-containing androgen receptor antagonist 3-(12hydroxymethyl-1,12-dicarba-closo-dodecaboran-1-yl) benzonitrile, Bioorg. Med. Chem., 2011, 19, 3540-3548.

9 A. Favila, M. Gallo and D. Glossman-Mitnik, CHIH-DFT determination of the molecular structure infrared spectra, UV spectra and chemical reactivity of three antitubercular compounds: Rifampicin, Isoniazid and Pyrazinamide, $J$. Mol. Model., 2007, 13, 505-518.

10 H. M. Badawi and W. Foerner, Analysis of the molecular structure and vibrational spectra of the indole based analgesic drug indomethacin, Spectrochim. Acta, Part A, 2014, 123, 447-454.

11 K. Moriyama, N. Furuno and N. Yamakawa, Crystal face identification by Raman microscopy for assessment of crystal habit of a drug, Int. J. Pharm., 2015, 480, 101-106.

12 D. Padula, B. L. Di, F. Santoro, H. Gerlach and A. Rizzo, Analysis of the electronic circular dichroism spectrum of (-)-[9](2,5)Pyridinophane, Chirality, 2012, 24, 994-1004.

13 M. M. Knopp, K. Lobmann, D. P. Elder, T. Rades and R. Holm, Recent advances and potential applications of modulated differential scanning calorimetry (mDSC) in drug development, Eur. J. Pharm. Sci., 2016, 87, 164-173. 
14 S. T. Ulu and F. T. Elmali, Highly sensitive spectrophotometric methods for the determination, validation and thermogravimetric analysis of antiulcer drugs, nizatidine and ranitidine in pure and pharmaceutical preparations, J. Anal. Chem., 2013, 68, 495500.

15 E. P. Lavor, M. V. M. Navarro, F. D. Freire, C. F. S. Aragao, F. N. Raffin, E. G. Barbosa and T. F. A. de Lima e Moura, Application of thermal analysis to the study of antituberculosis drugs-excipient compatibility, J. Therm. Anal. Calorim., 2014, 115, 2303-2309.

16 C. Dahlberg, S. V. Dvinskikh, M. Schuleit and I. Furo, Polymer Swelling, Drug Mobilization and Drug Recrystallization in Hydrating Solid Dispersion Tablets Studied by Multinuclear NMR Microimaging and Spectroscopy, Mol. Pharm., 2011, 8, 1247-1256.

17 A. Butykai, A. Orban, V. Kocsis, D. Szaller, S. Bordacs, E. Tatrai-Szekeres, L. F. Kiss, A. Bota, B. G. Vertessy, T. Zelles and I. Kezsmarki, Malaria pigment crystals as magnetic micro-rotors: key for high-sensitivity diagnosis, Sci. Rep., 2013, 3, 1431.

18 A. Watanabe, Study of crystalline drugs means of a polarizing microscope - XIX - A trial production of a table of the optical crystallographic characteristics of crystalline drugs including crystal habits, Yakugaku Zasshi, 2002, 122, 595-606.

19 S. R. Byrn, Solid-state chemistry of drugs, 2nd edn, Ssci Inc, West Lafayette, 1999.

20 R. Jenkins and R. Snyder, An introduction to X-Ray powder diffractometry, 1st edn, John Wiley \& Sons Inc, New York, 1996.

$21 \mathrm{~J} . \mathrm{Xu}, \mathrm{V} . \mathrm{V}$. Terskikh, Y. Chu, A. Zheng and Y. Huang, Mapping Out Chemically Similar, Crystallographically Nonequivalent Hydrogen Sites in Metal-Organic Frameworks by $1 \mathrm{H}$ Solid-State NMR Spectroscopy, Chem. Mater., 2015, 27, 3306-3316.

22 Y. Du, H. Zhang, J. Xue, H. Fang, Q. Zhang, Y. Xia, Y. Li and Z. Hong, Raman and terahertz spectroscopical investigation of cocrystal formation process of piracetam and 3hydroxybenzoic acid, Spectrochim. Acta, Part A, 2015, 139, 488-494.

23 M. Tonouchi, Cutting-edge terahertz technology, Nat. Photonics, 2007, 1, 97-105.

24 P. H. Siegel, Terahertz technology, IEEE Trans. Microwave Theory Tech., 2002, 50, 910-928.

25 C. J. Strachan, P. F. Taday, D. A. Newnham, K. C. Gordon, J. A. Zeitler, M. Pepper and T. Rades, Using terahertz pulsed spectroscopy to quantify pharmaceutical polymorphism and crystallinity, J. Pharm. Sci., 2005, 94, 837-846.

26 D. G. Allis, A. M. Fedor, T. M. Korter, J. E. Bjarnason and E. R. Brown, Assignment of the lowest-lying $\mathrm{THz}$ absorption signatures in biotin and lactose monohydrate by solid-state density functional theory, Chem. Phys. Lett., 2007, 440, 203-209.

27 H.-B. Liu, Y. Chen and X. C. Zhang, Characterization of anhydrous and hydrated pharmaceutical materials with
THz time-domain spectroscopy, J. Pharm. Sci., 2007, 96, 927-934.

28 H.-B. Liu and X. C. Zhang, Dehydration kinetics of D-glucose monohydrate studied using $\mathrm{THz}$ time-domain spectroscopy, Chem. Phys. Lett., 2006, 429, 229-233.

29 L. A. Sterczewski, M. P. Grzelczak, K. Nowak, B. Szlachetko and E. F. Plinski, Bayesian separation algorithm of $\mathrm{THz}$ spectral sources applied to D-glucose monohydrate dehydration kinetics, Chem. Phys. Lett., 2016, 644, 45-50.

30 J. A. Zeitler, K. Kogermann, J. Rantanen, T. Rades, P. F. Taday, M. Pepper, J. Aaltonen and C. J. Strachan, Drug hydrate systems and dehydration processes studied by terahertz pulsed spectroscopy, Int. J. Pharm., 2007, 334, 78-84.

31 J. Yang, S. Li, H. Zhao, B. Song, G. Zhang, J. Zhang, Y. Zhu and J. Han, Molecular Recognition and Interaction between Uracil and Urea in Solid-State Studied by Terahertz Time-Domain Spectroscopy, J. Phys. Chem. A, 2014, 118, 10927-10933.

32 Y. Du, Y. Xia, H. Zhang and Z. Hong, Using terahertz timedomain spectroscopical technique to monitor cocrystal formation between piracetam and 2,5-dihydroxybenzoic acid, Spectrochim. Acta, Part A, 2013, 111, 192-195.

33 K. L. Nguyen, T. Friscic, G. M. Day, L. F. Gladden and W. Jones, Terahertz time-domain spectroscopy and the quantitative monitoring of mechanochemical cocrystal formation, Nat. Mater., 2007, 6, 206-209.

34 Y. J. Son, D.-K. Lee and J.-H. Son, Identification of interpolymorph transformations of progesterone by terahertz time-domain spectroscopy, Curr. Appl. Phys., 2016, 16, 45-50.

35 T. Shibata, T. Mori and S. Kojima, Low-frequency vibrational properties of crystalline and glassy indomethacin probed by terahertz time-domain spectroscopy and low-frequency Raman scattering, Spectrochim. Acta, Part A, 2015, 150, 207-211.

36 S. Yamaguchi, K. Tominaga and S. Saito, Intermolecular vibrational mode of the benzoic acid dimer in solution observed by terahertz time-domain spectroscopy, Phys. Chem. Chem. Phys., 2011, 13, 14742-14749.

37 M. Walther, P. Plochocka, B. Fischer, H. Helm and P. U. Jepsen, Collective vibrational modes in biological molecules investigated by terahertz time-domain spectroscopy, Biopolymers, 2002, 67, 310-313.

38 B. Ferguson and X. C. Zhang, Materials for terahertz science and technology, Nat. Mater., 2002, 1, 26-33.

39 H. B. Liu and X. C. Zhang, Terahertz Spectroscopy for Explosive, Pharmaceutical, and Biological Sensing Applications, Springer, Netherlands, 2007.

40 X. Yang, K. Yang, Y. Luo and W. Fu, Terahertz spectroscopy for bacterial detection: opportunities and challenges, Appl. Microbiol. Biotechnol., 2016, 100, 5289-5299.

41 A. G. Markelz, Terahertz Dielectric Sensitivity to Biomolecular Structure and Function, IEEE J. Sel. Top. Quantum Electron., 2008, 14, 180-190.

42 M. Yamaguchi, F. Miyamaru, K. Yamamoto, M. Tani and M. Hangyo, Terahertz absorption spectra of L-, D-, and 
DL-alanine and their application to determination of enantiometric composition, Appl. Phys. Lett., 2005, 86, 053903.

43 M. D. King, P. M. Hakey and T. M. Korter, Discrimination of Chiral Solids: A Terahertz Spectroscopic Investigation of Land DL-Serine, J. Phys. Chem. A, 2010, 114, 2945-2953.

44 Y. L. Shi and L. Wang, Collective vibrational spectra of alphaand gamma-glycine studied by terahertz and Raman spectroscopy, J. Phys. D: Appl. Phys., 2005, 38, 3741-3745.

45 J. T. K. And and C. A. Schmuttenmaer, Far-Infrared Dielectric Properties of Polar Liquids Probed by Femtosecond Terahertz Pulse Spectroscopy, J. Phys. Chem., 1996, 100, 10373-10379.

46 S. Yan, D. Wei, M. Tang, C. Shi, M. Zhang, Z. Yang, C. Du and H. L. Cui, Determination of Critical Micelle Concentrations of Surfactants by Terahertz Time-Domain Spectroscopy, IEEE Trans. Terahertz Sci. Technol., 2016, 6, 532-540.

47 P. J. Larkin, M. Dabros, B. Sarsfield, E. Chan, J. T. Carriere and B. C. Smith, Polymorph Characterization of Active Pharmaceutical Ingredients (APIs) Using Low-Frequency Raman Spectroscopy, Appl. Spectrosc., 2014, 68, 758-776.

48 P. J. Larkin, J. Wasylyk and M. Raglione, Application of Lowand Mid-Frequency Raman Spectroscopy to Characterize the
Amorphous-Crystalline Transformation of Indomethacin, Appl. Spectrosc., 2015, 69, 1217-1228.

49 O. Daisuke, O. Yoshimichi and M. Maya, Crystallinity of poly(ethylene naphthalate) and its relation to terahertz absorption, Jpn. J. Appl. Phys., 2017, 56, 032402.

$50 \mathrm{Y}$. Xu and M. Havenith, Perspective: Watching low-frequency vibrations of water in biomolecular recognition by $\mathrm{THz}$ spectroscopy, J. Chem. Phys., 2015, 143, 170901.

51 S. Wietzke, C. Jansen, T. Jung, M. Reuter, B. Baudrit, M. Bastian, S. Chatterjee and M. Koch, Terahertz timedomain spectroscopy as a tool to monitor the glass transition in polymers, Opt. Express, 2009, 17, 19006-19014.

52 J. Qin, L. Xie and Y. Ying, Feasibility of Terahertz TimeDomain Spectroscopy to Detect Tetracyclines Hydrochloride in Infant Milk Powder, Anal. Chem., 2014, 86, 11750-11757.

53 J. B. Sleiman, B. Bousquet, N. Palka and P. Mounaix, Quantitative Analysis of Hexahydro-1,3,5-trinitro-1,3,5, Triazine/Pentaerythritol Tetranitrate (RDX-PETN) Mixtures by Terahertz Time Domain Spectroscopy, Appl. Spectrosc., 2015, 69, 1464-1471. 